\title{
Assessment of Creative Thinking of Hong Kong Undergraduate Students Using the Torrance Tests of Creative Thinking
}

\section{Annie W.Y. Ng, Chung-Yee Lee}

Office of Institutional Research, The Hong Kong University of Science and Technology, Hong Kong.

\begin{abstract}
Creativity has been a key graduate attribute and transferrable skill for the universities nowadays. A better understanding of freshmen students' level of creativity thinking facilitates the cultivation of the ways for nurturing the development of creativity in students throughout their university education studies. The purpose of this study was to investigate creative thinking abilities of first-year undergraduates in Hong Kong using the Torrance Tests of Creativity Thinking (TTCT) - a commonly known assessment for creativity. A total of 492 first-year undergraduates at a university in Hong Kong were asked to complete TTCT Figural Form consisting of picture construction, picture completion and lines activities. Generally, the creative potential of Hong Kong undergraduates was well above the average. The students demonstrated their abilities in generation of a number of relevant ideas (fluency), producing novel responses (originality), abstract thinking (abstractness of titles), and open-minded thinking (premature closure). They exhibited creative strength of, for example, flexible imagination, thinking with senses, and thinking beyond boundaries. However, students' elaborative thinking, metaphorical-thinking and sense of humor had a lot of room for improvement. Based on the assessment results, some implications on the possible institutional strategies for nurturing creativity among students during university education were discussed.
\end{abstract}

Keywords: Creativity; assessment; higher education; institutional research . 


\section{Introduction}

In Hong Kong, the development of creativity of students becomes one of the objectives in higher education for the 21st century, based on the latest trends and demands of society's needs (Education Commission, 2000; 2001). There are numerous fastest-growing jobs and emerging industries relying on creative capacity of employees to spot new and unforeseen business opportunities. Developing, implementing and communicating new ideas to others; and being open and responsive to new and diverse perspective are typical necessary creative and innovation skills at work nowsadays (Piirto, 2011). Such employment market tendencies highlgiht the need for university institutions to develop and underand the abilities of their students in creative thinking. Creativity thus acts as a key graduate attribute and transferrable skill for the four-year degree education in universities.

Measuring creative thinking abilities of students is increasing in education in general (Bart et al., 2017). The Torrance Tests of Creativity Thinking (TTCT) is a widely used assessment for creativity in different cultures and populations (e.g. Higuchi et al., 2013; Kim, 2017; Runco et al., 2010; Van Goch, 2018; Yarbrough, 2016). It has also been utilized for research purposes in Hong Kong with school children (Kam, 2009; Rudowicz et al. 1995). The TTCT was determined as the significance measure of creative potential of individuals, as its construct validity, convergent validity, discriminant validity, predictive validity, and test-retest reliability were comprehensively examined in prior studies (Kim et al., 2006; Kim, 2011; Yoon et al. 2017). The creative thinking abilities as measured in the TTCT referred to the "constellation of generalized mental abilities that are commonly presumed to be brought into play in creative achievements" (Scholastic Testing Service, 2017a).

A thorough understanding of freshmen students' level of creativity would help to better point out the ways for facilitating and fostering the development of creativity amongst the students during their university education. Taking Hong Kong as a cases study, the purpose of this study was to examine the degree of creative thinking abilities of first-year undergraduates as measured by the TTCT. The creativity measures of the Hong Kong sample were then compared with those from the norms. Based on the assessment results, some implications on the educational strategies for fostering creativity in students were discussed. The findings of this study would be a useful reference for instructional planning and setting out important curricular elements related to creativity in university programs according to the creative potential of intake undergraduates. 


\section{Method}

\subsection{Participants}

A total of 492 first-year undergraduate students at a university in Hong Kong voluntarily participated in this study. The mean age of the students was 17.91 years ( $\mathrm{SD}=0.68$ years). The proportions of males $(50.20 \%)$ to females $(49.80 \%)$ were approximately equal. Around three quarters of the participants (74\%) were local Hong Kong Chinese, and the remaining $26 \%$ participants were from other regional areas. The participants came from different disciplines including business, engineering, science, and social science.

\subsection{Instruments}

Torrance Tests of Creative Thinking (TTCT) Figural Form A consisting of three activities of picture construction, picture completion and lines was used in this study. The responses to the TTCT were drawings. The Figural Response Booklet A, a soft black lead pencil and a rubber were provided for the participants to complete the three activites.

\subsection{Procedure}

The Figural Form A was administered to the participants following the TTCT Directions Manual (Torrance, 2016). The participants were asked to complete the picture construction, followed by the picture completion and then the lines activity; each of which was completed in 10 minutes. The picture construction task required drawing of a picture based on a curved shape and creation of a title for the drawing. The picture completion task demanded the participants to complete 10 figures that appeared as incomplete and to give titles for the figures. The lines task asked the participants to draw as many figures as possible from the pairs of straight lines and to add titles for the figures.

\subsection{Measures}

The test responses of the participants were sent to the publisher of the TTCT for scoring. The Streamlined Scoring Guide (Scholastic Testing Service, 2017b) provided the details of the scoring mechanism. Two composite measures of 'grade-based average standard score' and 'grade-based creativity index' and six subscales of fluency, originality, elaboration, abstractness of titles, resistance of premature closure, and creative strength were adopted in this study. Fluency, originality, elaboration, abstractness of titles, and resistance of premature closure were called creativity dimensions (Table 1). Creative strength referred to the thirteen criterion-referenced measures such as emotional expressiveness, storytelling articulateness, extending or breaking boundaries, and richness of imagery (Table 2). 
Table 1. Five TTCT creativity dimensions (Scholastic Testing Service, 2017b).

\section{Dimension}

Fluency

Originality

Elaboration

Abstractness of title

Resistance of premature closure

\section{Description}

Ability to generate large number of relevant responses

Ability to produce novel or unusual responses

Ability to produce new response by adding tweaks to existing ideas

Ability to go beyond a concrete physcial description

Ability to keep open-minded and refrain from making premature conclusion

Table 2. The thirteen aspects of creative strength (CS) (Scholastic Testing Service, 2017b).

Item

CS1. Emotional expressiveness

CS2. Storytelling articulateness

CS3. Movement or action

CS4. Expressiveness of titles

CS5. Synthesis of incomplete figures

CS6. Synthesis of lines

CS7. Unusual visualization

CS8. Internal visualization

CS9. Extending or breaking boundaries

CS10. Humor

CS11. Richness of imagery

CS12. Colorfulness of imagery

CS13. Fantasy

\section{Description}

Feelings and emotions communicated through titles and drawings

Putting things in context and creating an environment for an object

Indicators of movement such as running, dancing and falling

Titles that go beyond simple description

Combination of two or more incomplete figures

Combination of two or more sets of lines

Visual perspective other than the static, upright, straight-on view

Objects with visible interior, cross-section, etc.

Going beyond the limits or boundaries of the parallel lines

Portraying something comical, funny or amusing

Responses showing variety, vividness, liveliness, intensity, etc.

Responses exicting to the senses, emotionally appealing, unreal, etc.

Figures in myths, fairy tales, science fiction, etc. 


\section{Results}

\subsection{Composite measures and five creativity dimensions}

The standard scores for each of the measures of creativity assessed by the TTCT were used in this study. The standard score was a normalized standard score reported on a scale with a mean of 100 and a standard deviation of 20 (Scholastic Testing Service, 2017b). The composite measures of creativity index and average standard score of the students were 116.30 and 102.02 respectively. The five creativity dimensions were examined for studying specific creativity-relevant skills. Among the five creativity dimensions, the mean of originality was highest (108.05), while elaboration was the lowest (89.97). The mean scores for fluency, abstractness of titles, and resistance of premature closure were 104.14, 102.05, and 105.84, respectively. The standard deviation on the creativity dimensions showed that individual difference of each dimension was in the following descending order: abstractness of titles (20.42), fluency (19.98), resistance of premature closure (19.48), originality (19.07), and elaboration (16.88).

\subsection{Creative Strength}

The classification levels of creative strength (CS) aspects were also examined in this study. Regarding items CS1 (Emotional expressiveness), CS4 (Expressiveness of titles), CS8 (Internal visualization), and CS13 (Fantasy), 45\%-51\% of the students indicated level of some evidences of these strength aspects.

For items CS2 (Storytelling articulateness; 75.81\%), CS3 (Movement of action; 87.20\%), CS9 (Extending or breaking boundaries; 77.24\%), and CS12 (Colorfulness of imagery; $80.28 \%$ ), more than three quarters of the students in this study showed repeated evidence of these strength aspects. For items CS7 (Unusual visualization) and CS11 (Richness of imagery), $50.61 \%$ and $40.04 \%$ of the students demonstrated repeated evidence respectively.

However, for items CS5 (Synthesis of incomplete figures; 90.24\%), CS6 (Synthesis of lines; 88.62\%), and CS10 (Humor; 70.53\%), most of the students showed absence of evidence of these creative strength aspects in the TTCT.

\subsection{Comparison with norm group}

A comparison was made between the Hong Kong results and those from the norms of the TTCT. The normative sample consisted of 1,936 American students at or above Grade 13 (Scholastic Testing Service, 2017a). The creativity index, average standard score, creativity dimensions, and creative strength were comparable to norm percentiles which informed the participant's rank compared to the norm average (mean percentile, $\mathrm{M}=50$ ). Participants' creativity Index $(M=54.49 ; \mathrm{SD}=28.15)$ and average standard score $(\mathrm{M}=54.49, \mathrm{SD}=28.15)$ were above average of the norm group. Of the five creativity dimensions, the scores for 
fluency $(M=56.32, S D=29.12)$, originality $(M=61.66, S D=27.71)$, abstractness of titles $(\mathrm{M}=53.13, \mathrm{SD}=29.53)$, and resistance of premature closure $(\mathrm{M}=58.36, \mathrm{SD}=28.52)$ were above average, but the score for elaboration was particularly low $(\mathrm{M}=35.06, \mathrm{SD}=24.78)$ as compared to the norm group.

\section{Discussion}

The creative potential of Hong Kong undergraduates was generally well above the average. This study revealed that the students tended to be good at producing a large number of relevant ideas (fluency dimension) and uncommon responses (originality dimension); abstract thinking (abstractness of titles dimension); and open-minded thinking (premature closure dimension). However, the capacity of students to expand and enrich existing ideas (elaboration dimension) was not good as compared to the other four dimensions.

With respective to the creative strength, this study showed that the Hong Kong undergraduate students demonstrated some evidences in flexible imagination - generation of ideas from different angles and perceiving a common object or situation in different ways (internal visualization and unusual visualization), and disregarding exist norms in unrealistic thoughts (fantasy) (Kim, 2017). The students also exhibited their abilities in thinking beyond the limits or boundaries (extending or breaking boundaries), thinking with senses such as sight, sound, smell, taste, touch and feel (colorfulness of imagery and emotional expressiveness), thinking with movement or action, creating vividness and sharp ideas with a lot of details (richness of imagery), and storytelling articulateness.

However, the Hong Kong undergraduate students lacked of capability to recognize relationships among rather diverse and unrelated elements (synthesis of incomplete figures), and capability to identify possibilities that majority of people assume have been closed and under restrictive conditions (synthesis of lines). This indicated that the students were generally absent of evidence of the creative strength of metaphorical-thinking for bridging conceptual gaps to view ideas from new perspectives (Kim, 2017). Rudowicz and Yue (2000) revealed that humorous was not in Chinese conceptions of creativity. In this study, the Hong Kong undergraduate student sample which was dominant with Chinese was found to be deficiency of sense of humor - the quality of portraying their works and ideas comical, funny, playful and amusing.

Creative thinking has been placed at the core of knowledge economy and most work these days with significant economic value requires creativity. The findings of creative potential of first-year undergraduate students in this study indicated that administrators, educators and all other practitioners should further develop students' creative abilities in particular in elaborative, metaphorical and playful thinking throughout their university education. For example, (i) practitioners teach creativity directly by equipping students with relevant 
creative thinking models and techniques to improve creative thinking skills in curricula and co-curricular programs. (ii) Institutions can help students develop their competence for creativity through teaching strategies such as cooperative learning which was found to enhance elaborative thinking of students (Palmer et al., 2016). (iii) Institutions should also put more effort to cultivate a culture of creativity on campus and nurture a creative learning environment. This would allow students to be free from the pressure and fear of failure during creative process, and be comfortable in trying to explore possibilities, experiment with amusing ideas and witness creativity in action.

\section{Conclusion}

This study examined the creative thinking abilities of first-year undergraduates in Hong Kong using a commonly known assessment for creativity, the Torrance Tests of Creativity Thinking (TTCT). The creative potential of Hong Kong undergraduates, in general, was well above the average. The students showed their capabilities in generation of a number of relevant ideas (fluency), producing novel responses (originality), abstract thinking (abstractness of titles), and open-minded thinking (premature closure). They also demonstrated creative strength of flexible imagination, disregarding exist norms, thinking beyond boundaries, thinking with senses, richness of imagery, and storytelling articulateness. The elaborative thinking, metaphorical-thinking and sense of humor of the students, however, needed to be enhanced. Implications on the possible strategies for nurturing creativity in students were discussed, and the strategies consisted of three main areas namely creative atmosphere of institution, curriculum design and teaching methodologies. The results and implications of this assessment would become a reference for administrators and educators to develop students' creativity during university education.

\section{References}

Bart, W. M., Hokanson, B., \& Can, I. (2017). An investigation of the factor structure of the Torrance Tests of Creative Thinking. Educational Sciences: Theory \& Practice, 17, $515-528$.

Education Commission. (2000). Learning for Life, Learning through Life - Reform Proposals for the Education System in Hong Kong. Hong Kong. Retrieved from

https://www.e-c.edu.hk/en/publications_and_related_documents/rf1.html

Education Commission. (2001). Reform of the Education System in Hong Kong - Summary. Hong Kong. Retrieved from https://www.e-c.edu.hk/en/publications_and_related_documents/rf2.html

Higuchi, T., Yuizono, T., Miyata, K., Sakurai, K., \& Kawaji, T. (2013). Creativity effects of idea-marathon system (IMS): Torrance Tests of Creative Thinking (TTCT) figural 
tests for college atudents. In A. M. J. Skulimowski (Ed.), Proceedings of KICSS'2013 (pp. 138-148). Kraków: Progress \& Business Publishers.

Kam, S. W. S. (2009). An Examination of the Creativity of Young Children in Four Child Care Centres in Hong Kong (Doctoral dissertation). Australia: University of South Australia.

Kim, K. H. (2011). The APA 2009 division 10 debate: are the Torrance Tests of Creative Thinking still relevant in the 21st century? Psychology of Aesthetics, Creativity, and the Arts, 5(4), 302-308.

Kim, K. H. (2017). The Torrance Test of Creative Thinking - figural or verbal: which one should we use? Creativity: Theories, Research and Applications, 4(2), 302-321.

Kim, K. H., Cramond, B., \& Bandalos, D. L. (2006). The latent structure and measurement invariance of scores on the Torrance Tests of Creative Thinking - figural. Educational and Psychological Measurement, 66(3), 459-477.

Palmer, G., Peters, R., Streetman, R. (2016). Cooperative Learning. In P. Lombardi (Ed.), Instructional Methods, Strategies and Technologies to Meet the Needs of All Learners (p.111-136). U.S.:Granite State College. Retrieved from

https://granite.pressbooks.pub/teachingdiverselearners/

Piirto, J. (2011). Creativity for 21st Century Skills: How to Embed Creativity into the Curriculum. Rotterdam/Boston/Taipei: Sense Publishers.

Rudowicz, E., Lok, D., \& Kitto, J. (1995). Use of the Torrance Tests of Creative Thinking in an exploratory study of creativity in Hong Kong primary school children: a crosscultural comparison. International Journal of Psychology, 30(4), 417-430.

Rudowicz, E., \& Yue, X. D. (2000). Concepts of creativity: similarities and differences among Mainland, Hong Kong and Taiwanese children. Journal of Creative Behavior, 34(3), 175-192.

Runco, M. A., Miller, G., Acar, S., \& Cramond, B. (2010). Torrance Tests of Creative Thinking as predictors of personal and public achievement: a fifty-year follow-up. Creativity Research Journal, 22(4), 361-368.

Scholastic Testing Service (2017a). Streamlined Scoring Guide for Figural Forms A and B. Illinois, U.S.A.

Scholastic Testing Service (2017b). Norms-Technical Manual for Figural Forms A and B. Illinois, U.S.A.

Torrance, E. P. (2016). Torrance Tests of Creative Thinking Directions Manual Figural Forms $A$ and B. Scholastic Testing Service: Illinois, U.S.A.

Van Goch, M. (2018). Creativity in liberal education before and after study commencement. In J. Domenech, P. Merello, E. de la Poza, D. Blazquez (Eds.), Proceedings of 4th International Conference on Higher Education Advances (HEAd' 18) (pp. 1475-1483). València, Spain: Universitat Politecnica de València. doi: 10.4995/HEAd18.2018.8228.

Yarbrough, N. D. (2016). Assessment of creative thinking across cultures using the Torrance Tests of Creative Thinking (TTCT): translation and validity Issues. Creativity Research Journal, 28(2), 154-164.

Yoon, C. H. (2017). A validation study of the Torrance Tests of Creative Thinking with a sample of Korean elementary school students. Thinking Skills and Creativity, 26, 38-50. 